\title{
Invasive mole of the uterus - a rare case diagnosed by ultrasound: a case report
}

\author{
Kavitha Nair, Hanaa Al-Khawari
}

Department of Radiology, Kuwait Cancer Control Center, Ministry of Health, Shuwaikh, Kuwait

\begin{abstract}
Gestational trophoblastic neoplasias (GTN) are rare tumours that constitute less than $1 \%$ of all gynecological malignancies. Invasive mole is a distinct subgroup of GTN, which if not diagnosed and treated early, can result in serious complications like uterine perforation and haemoperitoneum. We present a rare case of an invasive mole of the uterus, which developed following the evacuation of a molar pregnancy. It was accurately diagnosed by transvaginal ultrasound and color Doppler, and successfully treated before any major complications could arise.

Keywords: complete hydatiform mole, invasive mole, beta-human chorionic gonadotropin, transvaginal ultrasound, color Doppler ultrasound
\end{abstract}

\section{Introduction}

Gestational Trophoblastic Disease (GTD) is an umbrella term for a group of pregnancy related disorders arising from abnormal placental trophoblast cells. It encompasses two pre-malignant conditions: partial and complete hydatidiform moles and the malignant Gestational Trophoblastic Neoplasias (GTN). GTNs are classified histologically into three distinct subgroups: choriocarcinoma destruens (invasive mole), choriocarcinoma (CC) and the very rare placental site trophoblastic tumor (PSTT) [1]. Invasive moles are responsible for most cases of localized GTN. Invasive mole is a condition where a molar pregnancy invades the wall of the uterus. It follows approximately 10 $15 \%$ of complete hydatidiform moles and $0.5 \%$ of patients with partial mole [2]. The imaging of choice is B-mode

Received 5.04.2014 Accepted 10.04.2014

Med Ultrason

2014, Vol. 16, No 2, 175-178

Corresponding author: Kavitha Nair MBBS, DMRD, DNB,

FRCR (London)

Department of Radiology

Kuwait Cancer Control Center,

Ministry of Health, P.O.Box 42262

70653Shuwaikh, Kuwait

Phone: 00965-97156606

E-mail: kavitha63@gmail.com ultrasonography and color Doppler. When ultrasonography corresponds with the classical clinical presentation, an accurate diagnosis is possible in a majority of cases. With early and accurate diagnosis, the cure rate is high [3].

\section{Case report}

A 24 year old lady, gravida 2, para 1, presented to our department with irregular vaginal bleeding since 2 months and elevated beta-human chorionic gonadotropin (bHCG) level. Her physical examination was unremarkable. Her gynecological history was notable for the occurrence, 2 1/2 months ago, of an abortion at 12 weeks gestation, which turned out to be a complete molar pregnancy. She subsequently underwent a dilatation and curettage as the bHCG was persistently elevated $(10,000$ $\mathrm{IU} / \mathrm{ml}$ ). Following this, however, the bHCG still continued to rise, reaching up to $37,204 \mathrm{IU} / \mathrm{ml}$, as a result of which she underwent a second dilatation and curettage which was around 1 month prior presentation. This did not reveal any residual lesion in the endometrial cavity. A transvaginal ultrasound scan done at that time was reported as normal. She was subsequently followed up by serial bHCG levels, which though initially it fell to 3489 $\mathrm{IU} / \mathrm{ml}$, again started rising, reaching a level of $4613 \mathrm{IU} /$ $\mathrm{ml}$ at which time she was referred to our hospital. 


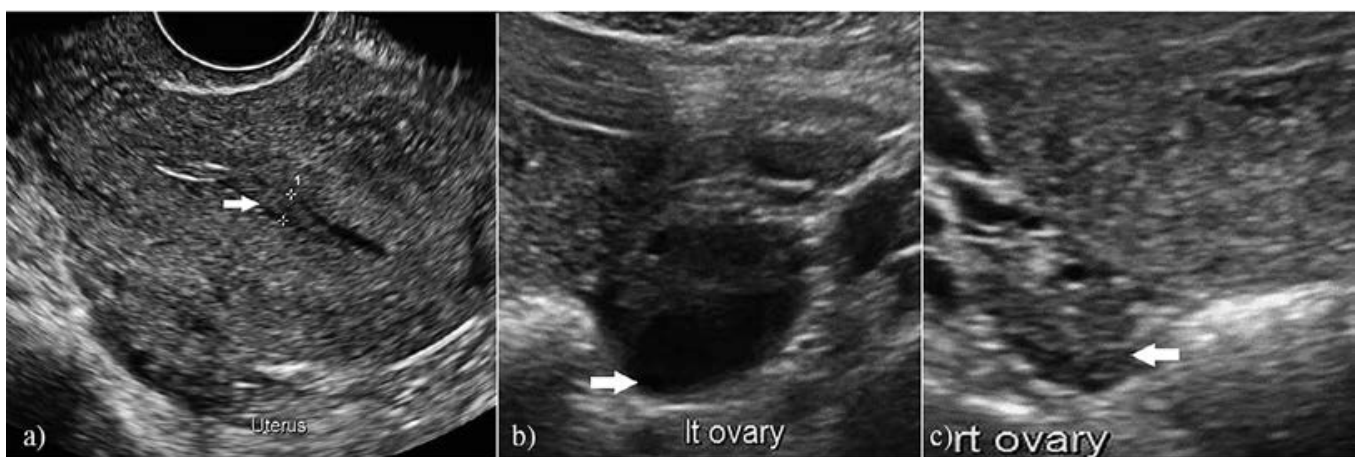

Fig 1. a) Transvaginal ultrasound demonstrated bulky uterus with echogenic fluid seen within the endometrial cavity (white arrow); b) Left ovary showed a simple anechoic cyst, measuring $2.3 \times 1.5 \mathrm{~cm}$ (white arrow); c) Right ovary (white arrow) was unremarkable.

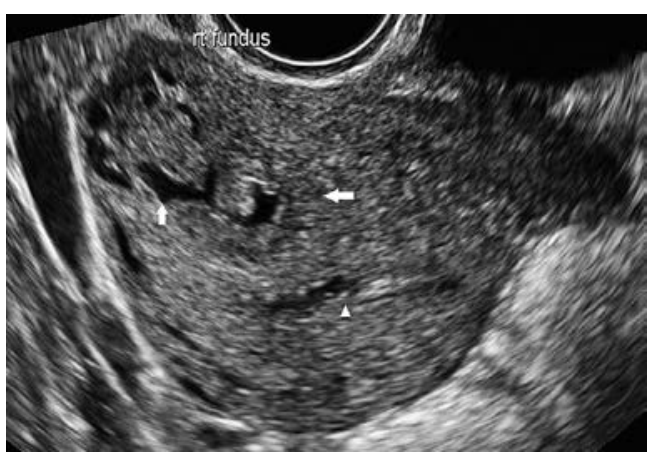

Fig 2. Transvaginal ultrasound showed an ill defined heterogeneous hyper echoic solid lesion (white arrow pointing to left) with cystic areas (white arrow pointing up) seen in the myometrium, right fundal region of the uterus, away from the endometrium (white arrowhead).

A transvaginal ultrasound scan revealed a bulky uterus with minimal fluid of mixed echogenicity suggestive of hemorrhage within the endometrial cavity. The left ovary showed a $2.3 \times 1.5 \mathrm{~cm}$ anechoic cyst.The right ovary was unremarkable (fig 1). A $3 \times 3 \times 1.8 \mathrm{~cm}$ ill defined heterogenous hyperechoic solid lesion with cystic vascular areas within it, was noted within the myometrium in the right fundal region of the uterus, away from the endometrium (fig 2). Colour Doppler showed prominent blood flow signals of various directions within the lesion, which were also seen within the cystic areas of the lesion. Waveform analysis showed low vascular impedance arterial blood flow (fig 3) as well as non pulsatile venous flow. No evidence of an intrauterine gestational sac or ectopic pregnancy was noted. Based on the clinical history of molar pregnancy in the past, with persistently elevated bHCG levels, a sonographic diagnosis of an invasive mole of the uterus was established. A complete metastatic work-up was done which did not reveal any abnormality.

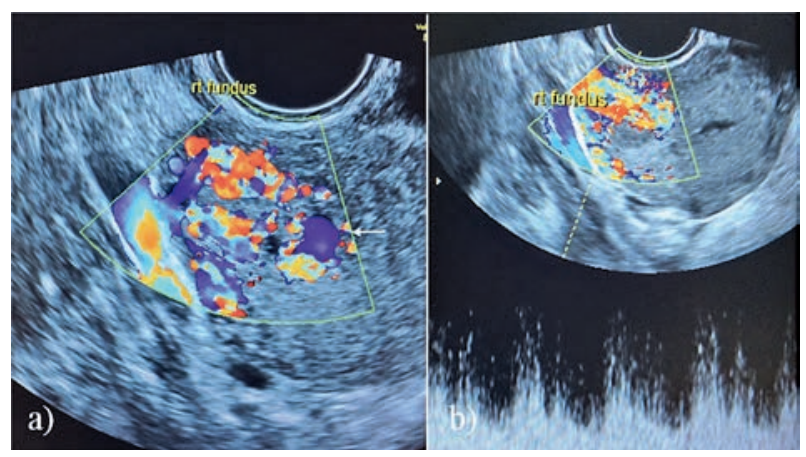

Fig 3. a) Colour Doppler showed prominent blood flow signals within the lesion (white arrow) suggestive of neovascularisation (vascularity was seen in the cystic areas as well); b) Pulsed Doppler showed low vascular impedance arterial blood flow.

The patient was started on Methotrexate immediately, and 2 weeks later, the bHCG showed a dramatic fall to $164 \mathrm{IU} / \mathrm{ml}$. After several cycles of chemotherapy, the bHCG reached a normal level of $0.7 \mathrm{IU} / \mathrm{ml}$. A repeat transvaginal ultrasound and color Doppler scan was done three months later, which did not reveal any abnormality, suggestive of complete response to therapy.

\section{Discussions}

GTN arises when the normal regulatory mechanisms controlling the proliferation and invasiveness of trophoblastic tissue are lost. Originating in placental tissue, they are characterized by a distinct tumour marker (bHCG) and have varying tendencies towards local invasion and distant metastasis [4].

In contrast to a hydatidiform mole (an intracavitary uterine lesion), the invasive hydatidiform mole, the placental site trophoblastic tumor, and the choriocarcinoma 
either invade or may be located within the myometrium. An invasive hydatiform mole is a form of GTN that occurs due to abnormal proliferation of placental trophoblast. It most commonly occurs after the evacuation of GTD. It is characterized by the presence of edematous chorionic villi with trophoblastic proliferation that invades into the myometrium of the uterus or to adjacent structures like the vagina, vulva, broad ligament, and can also invade into the uterine vessels [5]. Invasive mole is unlike choriocarcinoma, the latter is without the presence of chorionic villi. It is important to distinguish between invasive mole and choriocarcinoma, as the former has a more favorable outcome.

The clinical presentation of an invasive mole includes vaginal bleeding, an enlarged uterus and high urinary or serum bHCG level, typically after the evacuation of a molar pregnancy. The interval from an antecedent molar pregnancy is usually less than six months. Choriocarcinoma can occur after a hydatiform mole or even after a normal pregnancy, with an interval of more than six months, sometimes lasting for nearly ten years. BHCG levels are much higher in choriocarcinoma than in invasive mole [6].

Approximately $8 \%$ of patients with complete moles will develop a malignant tumour after evacuation [7]. Therefore serial values of bHCG should be obtained after evacuation, which if persistently elevated, should raise the suspicion of GTN. The Cancer Committee of the International Federation of Gynaecologists and Obstreticians (FIGO) has established the following guidelines for the diagnosis of post molar gestational trophoblastic neoplasia [8].

Four values or more of bHCG plateaued over at least three weeks.

An increase in bHCG of $10 \%$ or greater for three or more values over at least 2 weeks.

The histological diagnosis of choriocarcinoma.

Persistence of bHCG six months after molar evacuation.

Ultrasound has become the standard protocol in aiding in diagnosis of suspected GTN [9]. B-mode ultrasound is useful in detecting the presence of abnormal uterine masses. Sonographically, an invasive hydatidiform mole, a placental site trophoblastic tumor, and choriocarcinoma typically exhibit a heterogenous, hyperechoic, solid mass with cystic vascular spaces, located within the myometrium $[10,11]$. Colour Doppler imaging aids in the assessment of angiogenesis and neovascularisation characteristic in these tumors, seen as prominent blood flow signals in various directions suggestive of arterial and venous flow [12]. Doppler velocimetric findings in patients with invasive moles, placental site trophoblastic tumors and choriocarcinoma are similar in that they all may exhibit low-impedance arterial flow and high velocity [10]. Bilateral enlarged ovaries due to theca lutein cysts may also be seen [6].

The differential diagnosis of highly vascular, intramural lesions of the myometrium seen by ultrasound includes arteriovenous malformation, gestational trophoblastic neoplasia, and interstitial pregnancy [13]. It is possible to distinguish between these entities, using a combination of both biochemical findings and ultrasound appearances. Demonstration of a vascular mass within the myometrium without evidence of fetal material, on ultrasonography, in the context of an elevated bHCG is highly suggestive of GTN [14].

The pathological diagnosis of an invasive mole is rarely made, because most cases are conservatively treated, without the need for hysterectomy. Invasive mole is rarely metastatic, if it does occurs, it is usually to the lungs. Metastases are much more common in choriocarcinoma, common sites being the lower genital tract, brain, liver, lung, kidney, and the gastrointestinal tract [15]. The absence of metastases helps to rule to choriocarcinoma.

The FIGO committee on Gynecological oncology has made recommendations for the metastatic work up of GTN, which includes a chest X-ray, liver CT when indicated or a whole body CT scan in patients with lung metastases, and a brain MRI (or CT) when there is a suspicion of cerebral metastases.

Management of an invasive mole includes treatment with chemotherapy as well as continued monitoring of bHCG. Patients with GTN should be followed with weekly quantitative bHCG levels until normal for three consecutive weeks, then monthly for 12 months [16]. Ultrasound and Color Doppler have also been shown to be an effective tool in predicting the resolution or persistence of GTN post treatment [17]. Dilatation and curettage is not recommended due to the risk of uterine perforation [5]. With methotrexate, complete remission is achieved in most non-metastatic and low risk cases [18]. Even in the presence of disseminated disease, most of the cases are amenable to treatment with almost $100 \%$ survival.

\section{References}

1. Ngan HY, Bender H, Benedet JL, Jones H, Montruccoli GC, Pecorelli S; FIGO Committee on Gynecologic Oncology. Gestational trophoblastic neoplasia, FIGO 2000 staging and classification. Int J Gynaecol Obstet 2003; 83 Suppl 1: $175-177$.

2. Hammond CB. Gestational trophoblastic neoplasms. In: Scott JR, DiSaia PJ, Spellacy WN (eds). Danforth's Ob- 
stetrics and Gynecology, 8th ed. Philadelphia: Lippincott Williams \& Wilkins 1999: 927-937.

3. Tie W, Tajnert K, Plavsic SK. Ultrasound imaging of gestational trophoblastic disease. DSJUOG 2013; 7: 105-112.

4. Moodley M, Tunkyi K, Moodley J. Gestational trophoblastic syndrome: an audit of 112 patients. A South African experience. Int J Gynecol Cancer 2003; 13: 234-239.

5. Soper JT, Mutch DG, Schink JC; American College of Obstetricians and Gynecologists. Diagnosis and treatment of gestational trophoblastic disease: ACOG Practice Bulletin No.53. Gynecol Oncol 2004; 93: 575-585.

6. Maeda K, Kurjak A, Varga G, Honemeyer U. Trophoblastic diseases. DSJUOG 2012; 6: 27-42.

7. Martonffy AI, Rindfleisch K, Lozeau AM, Potter B. First trimester complications. Prim Care 2012; 39: 71-82.

8. Goldstein DP, Berkowitz RS. Current management of gestational trophoblastic neoplasia. Hematol Oncol Clin North Am 2012; 26: 111-131.

9. Jauniaux E. Ultrasound diagnosis and follow up of gestational trophoblastic disease. Ultrasound Obstet Gynecol 1998; 11: 367-377.

10. Zhou Q, Lei XY, Xie Q, Cardoza JD. Sonographic and doppler imaging in the diagnosis and treatment of gestational trophoblastic disease: a 12-year experience. J Ultrasound Med 2005; 24: 15-24.

11. Caspi B, Elchalal U, Dgani R, Ben-Hur H, Rozenman D, Nissim F. Invasive mole and placental site trophoblastic tu- mor. Two entities of gestational trophoblastic disease with a common ultrasonographic appearance. J Ultrasound Med 1991; 10: 517-519.

12. Seckl MJ, Sebire NJ, Berkowitz RS. Gestational trophoblastic disease. Lancet 2010; 376: 717-729.

13. Timmerman D, Wauters J, Van Calenbergh S, et al. Color Doppler imaging is a valuable tool for the diagnosis and management of uterine vascular malformations. Ultrasound Obstet Gynecol 2003; 21: 570-577.

14. Amandeep K, Shashi G, Vikram M, Rajni G. Invasive mole presenting as acute abdomen. J\&K -India 2011; 13: 35-36.

15. Smith HO, Kohorn E, Cole LA. Choriocarcinoma and gestational trophoblastic disease. Obstet Gynecol Clin North Am 2005; 32: 661-684.

16. May T, Goldstein DP, Berkowitz RS. Current chemo therapeutic management of patients with gestational trophoblastic neoplasia. Chemother Res Pract 2011; 2011: 806256.

17. Zanetta G, Lissoni A, Colombo M, Marzola M, Cappellini A, Mangioni C. Detection of abnormal intrauterine vascularization by color Doppler imaging. A possible additional aid for the follow up of patients with gestational trophoblastic tumours. Ultrasound Obstet Gynecol 1996; 7: 3237.

18. Homeslay HD. Single agent therapy for nonmetastatic andlow risk gestational trophoblastic disease. J Reprod Med 1998; 43: 69-74. 